\title{
TRANSPORT DYNAMICS OF BROAD RESONANCES
}

Jörn Knoll, GSI, Darmstadt

J.Knoll@gsi.de; http://theory.gsi.ds

\begin{abstract}
The propagation of short life time particles with consequently broad mass width are discussed in the context of transport descriptions. In the first part some known properties of finite life time particles such as resonances are reviewed and discussed at the example of the $\rho$-meson. Grave deficiencies in some of the transport treatment of broad resonances are disclosed and quantified. The second part addresses the derivation of transport equations which permit to account for the damping width of the particles. Baym's $\Phi$-derivable method is used to derive a self-consistent and conserving scheme, which fulfils detailed balance relations even in the case of particles with broad mass distributions. For this scheme a conserved energy-momentum tensor can be constructed. Furthermore, a kinetic entropy can be derived which besides the standard quasi-particle part also includes contributions from fluctuations.
\end{abstract}

\section{Introduction and Prospects}

With the aim to describe the collision of two nuclei at intermediate or even high energies one is confronted with the fact that the dynamics has to include particles like the $\Delta^{33}$ or $\rho$-meson resonances with life-times of less than $2 \mathrm{fm} / \mathrm{c}$ or equivalently with damping rates above $100 \mathrm{MeV}$. Also the collision rates deduced from presently used transport codes are comparable in magnitude, whereas typical mean kinetic energies as given by the temperature range between 70 to $150 \mathrm{MeV}$ depending on beam energy. Thus, the damping width of most of the constituents in the system can no longer be treated as a perturbation.

As a consequence the mass spectra of the particles in dense matter are no longer sharp delta functions but rather acquire a width due to collisions and decays. The corresponding quantum propagators $G$ (Green's functions) are no longer the ones as in standard text books for fixed mass, but have to be folded over a spectral function $A(\epsilon, \vec{p})$ of finite width. One thus comes to a picture which unifies resonances which have already a decay width in vacuum with the "states" of particles in dense matter, which obtain a width due to collisions (collisional broadening). The theoretical concepts for a proper many body description in terms of a real time non equilibrium field theory have already been devised by Schwinger, Kadanoff, Baym and Keldysh [1] in the early sixties. First investigations of the quantum effects on the Boltzmann collision term were given Danielewicz [2], the principal conceptual problems on the level of quantum field theory were investigated by Landsmann [3], while applications which seriously include the finite width of the particles in transport descriptions were carried out only in recent times, e.g. 2, 2, 10] For resonances, e.g. the $\Delta^{33}$-resonance, it was natural to consider broad mass distributions and ad hoc recipes have been invented to include this in transport simulation models. However, many of these recipes are not correct as they violate some basic principles like detailed balance [4], and the description of resonances in dense matter has to be improved.

In this talk the transport dynamics of short life time particles are reviewed and discussed. In the first part some known properties of resonances are presented. These concern the equilibrium and low density (virial) limits. Some example discussions are given for the di-lepton spectrum resulting from the decay of $\rho$-mesons in a dense nuclear environment, both in thermal equilibrium and in a quasi-free scattering process. On the basis of this some deficiencies of presently used transport codes for the treatment of broad resonances are disclosed and quantified. They affect the di-lepton spectra already on a qualitative level and signal that the low mass side is grossly underestimated in the respective calculations. This motivates the question discussed in the second part, namely, how to come to a self-consistent, conserving and thermodynamically consistent transport description of particles with finite mass width. The conceptual starting point will be a formulation within the real-time nonequilibrium field theory. The derivation is based on and generalizes Baym's $\Phi$-functional method 
[11]. The first-order gradient approximation provides a set of coupled equations of time-irreversible generalized kinetic equations for the slowly varying space-time part of the phase-space distributions supplemented by retarded equations. The latter account for the fast micro-scale dynamics represented by the four-momentum part of the distributions. Functional methods permit to derive a conserved energy-momentum tensor which also includes corrections arising from fluctuations besides the standard quasi-particle terms. Memory effects [12-14] appearing in collision term diagrams of higher order as well as the formulation of a non-equilibrium kinetic entropy flow can also be addressed [14].

\section{Preliminaries}

The standard text-book transition rate in terms of Fermi's golden rule, e.g. for the photon radiation from some initial state $|i\rangle$ with occupation $n_{i}$ to final states $|f\rangle$

$$
W=\left.\left.\sum_{i f} n_{i}\left(1-n_{f}\right)\right|_{i} ^{f} \uparrow\right|^{2}\left(1+n_{\omega}\right) \delta\left(E_{i}-E_{f}-\omega_{\vec{q}}\right)
$$

with occupation $n_{\omega}$ for the photon, is limited to the concept of asymptotic states. It is therefore inappropriate for problems which deal with particles of finite life time. One rather has to go to the "closed" diagram picture, where the same rate emerges as

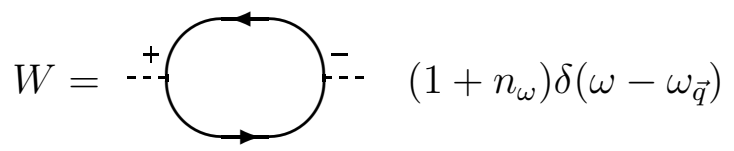

with now two types of vertices - and + for the time-ordered and the anti-time ordered parts of the square of the amplitude. Together with the orientation of the $\stackrel{+}{\longrightarrow}$ and $\stackrel{-}{\longrightarrow}$ propagator lines one obtains unique diagrammatic rules for the calculation of rates rather than amplitudes. The just mentioned propagator lines define the densities of occupied states or those of available states, respectively. Therefore all standard diagrammatic rules can be used again. One simply has to extend those rules to the two types of vertices with marks - and + and the corresponding 4 propagators, the usual time-ordered propagator $\stackrel{-}{\longrightarrow}$ between two - vertices, the anti-time-ordered one $\stackrel{++}{\longrightarrow}$ between two + vertices and the mixed $\stackrel{+}{\longrightarrow}$ or $\stackrel{-+}{\longrightarrow}$ ones with fixed operator ordering (Wightman-functions) as densities of occupied and available states. For details I refer to the textbook of Lifshitz and Pitaevski [15.

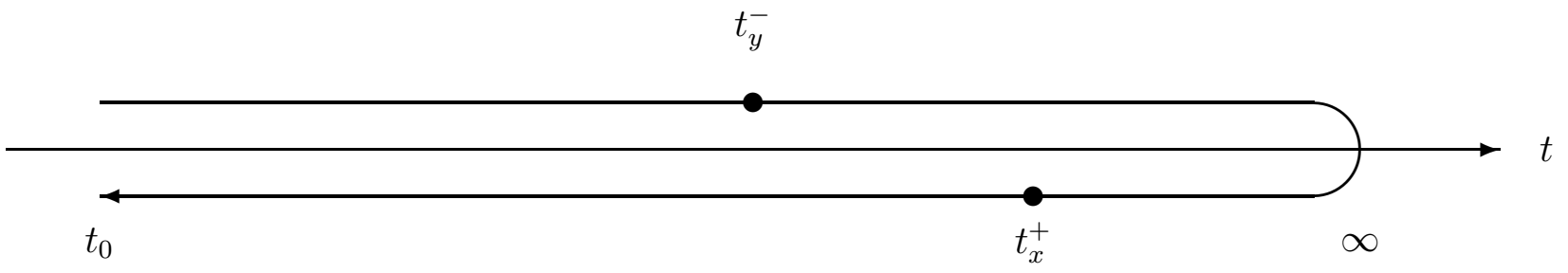

Fig. 1: Closed real-time contour with two external points $x, y$ on the contour.

Equivalently the non-equilibrium theory can entirely be formulated on one special time contour, the so called closed time path [1], fig. 1, with the time argument running from some initial time $t_{0}$ to infinity and back with external points placed on this contour, e.g., for the four different components of Green's functions or self energies. The special -+ or +- components of the self energies define the gain and loss terms in transport problems, c.f. eq. (2) and eqs. (23) 25) below.

The advantage of the formulation in terms of "correlation" diagrams, which no longer refer to amplitudes but directly to physical observables, like rates, is that now one is no longer restricted to the concept of asymptotic states. Rather all internal lines, also the ones which originally referred to the "in" or "out" states are now treated on equal footing. Therefore now one can deal with "states" which have a broad mass spectrum. The corresponding Wigner densities $\stackrel{+}{\longrightarrow}$ or $\stackrel{-+}{\longrightarrow}$ are then no longer 
on-shell $\delta$-functions in energy (on-mass shell) but rather acquire a width, as we shall discuss in more detail.

For slightly inhomogeneous and slowly evolving systems, the degrees of freedom can be subdivided into rapid and slow ones. Any kinetic approximation is essentially based on this assumption. Then for any two-point function $F(x, y)$, one separates the variable $\xi=\left(t_{1}-t_{2}, \overrightarrow{r_{1}}-\overrightarrow{r_{2}}\right)$, which relates to the rapid and short-ranged microscopic processes, and the variable $X=\frac{1}{2}\left(t_{1}+t_{2}, \overrightarrow{r_{1}}+\overrightarrow{r_{2}}\right)$, which refers to slow and long-ranged collective motions. The Wigner transformation, i.e. the Fourier transformation in four-space difference $\xi=x-y$ to four-momentum $p$ of the contour decomposed components of any two-point contour function

$$
F^{i j}(X ; p)=\int \mathrm{d} \xi e^{\mathrm{i} p \xi} F^{i j}(X+\xi / 2, X-\xi / 2), \quad \text { where } i, j \in\{-+\}
$$

leads to a (co-variant) four phase-space formulation of two-point functions. The Wigner transformation of Dyson's equation (19) in $\{-+\}$ notation is straight forward. For details and the extensions to include the coupling to classical field equations we refer to ref. [16].

Standard transport descriptions usually involve two approximation steps: (i) the gradient expansion for the slow degrees of freedom, as well as (ii) the quasi-particle approximation for rapid ones. We intend to avoid the latter approximation and will solely deal with the gradient approximation for slow collective motions by performing the gradient expansion of the coupled Dyson equations. This step indeed preserves all the invariances of the $\Phi$ functional in a $\Phi$-derivable approximation.

It is helpful to avoid all the imaginary factors inherent in the standard Green's function formulation and change to quantities which are real and positive either in the homogeneous or in appropriate coarse graining limits. They then have a straight physical interpretation analogously to the Boltzmann equation. We define

$$
\left.\begin{array}{l}
F(X, p)=A(X, p) f(X, p)=\mp \mathrm{i} G^{-+}(X, p), \\
\widetilde{F}(X, p)=A(X, p)[1 \mp f(X, p)]=\mathrm{i} G^{+-}(X, p),
\end{array}\right\} \quad \text { with } \quad A(X, p) \equiv-2 \operatorname{Im} G^{R}(X, p)=\widetilde{F} \pm F
$$

for the generalized Wigner functions $F$ and $\widetilde{F}$ with the corresponding four phase space distribution functions $f(X, p)$, the Fermi/Bose factors $[1 \mp f(X, p)]$ and spectral function $A(X, p)$. According to the retarded relations between Green's functions $G^{i j}$, only two of these real functions are required for a complete dynamical description. Here and below upper signs relate to fermion quantities, whereas lower signs refer to boson quantities. As shown in ref. [16] mean fields and condensates, i.e. non-vanishing expectation values of one-point functions can also be included.

\section{Thermodynamic Equilibrium}

The thermodynamic equilibrium leads to a lot of simplifying relations among the kinetic quantities. All quantities become space-time independent. The Kubo-Martin-Schwinger condition determines the distribution functions to be of Fermi-Dirac or Bose-Einstein type, respectively

$$
f_{\text {eq }}(X, p)=1 /\left(\exp \left(\left(p_{0}-\mu\right) / T\right) \pm 1\right)
$$

Here $\mu$ is the chemical potential. The spectral function attains a form

$$
A_{\text {eq }}(X, p)=\frac{\Gamma(p)}{M^{2}(p)+\Gamma^{2}(p) / 4} \quad \text { with } \quad\left\{\begin{aligned}
\Gamma(p)=-2 \operatorname{Im} \Sigma^{\mathrm{R}}(p), \\
M(p)=M_{0}(p)-\operatorname{Re} \Sigma^{\mathrm{R}}(p), \quad M_{0}(p)=p_{0}^{\kappa}-p_{0}^{\kappa}(\vec{p}) .
\end{aligned}\right.
$$

This form is exact through the four-momentum $p=\left(p_{0}, \vec{p}\right)$ dependence of the retarded self-energy $\Sigma^{\mathrm{R}}(p)$. Thereby $M_{0}(p)=p_{0}^{\kappa}-p_{0}^{\kappa}(\vec{p})=0$ is the free dispersion relation with $\kappa=1$ or 2 for the non-relativistic Schrödinger or the relativistic Klein-Gordon case, respectively. In the non-equilibrium case all quantities become functions of the space-time coordinates $X$ and, of course, the distribution functions $f(X, p)$ generally also depend on three momentum $\vec{p}$. 


\section{The Virial Limit}

Another simplifying case is provided by the low density limit, i.e. the virial limit. Since Beth-Uhlenbeck (1937) 17 it is known that the corrections to the level density are given by the asymptotic properties of binary scattering processes, i.e. in a partial wave decomposition by means of phase-shifts, see also [18-20]. The reasoning can be summarized as follows. While for any pair the c.m. motion remains unaltered the relative motion is affected by the mutual two-body interaction. Considering a large quantization volume of radius $R$ and a partial wave of angular momentum $j$, the levels follow the quantization condition

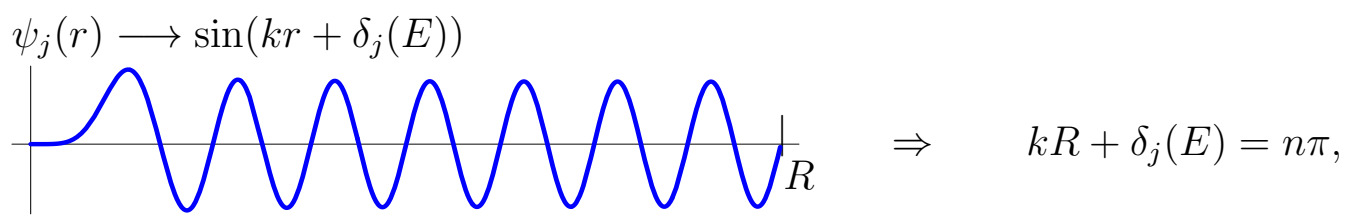

where $\delta_{j}(E)$ is the phase-shift at relative energy $E$ and $n$ is an integer counting the levels. The $k R$ term accounts for the free motion part. The corresponding corrections to both, the level density and thermodynamic partition sum, are given by

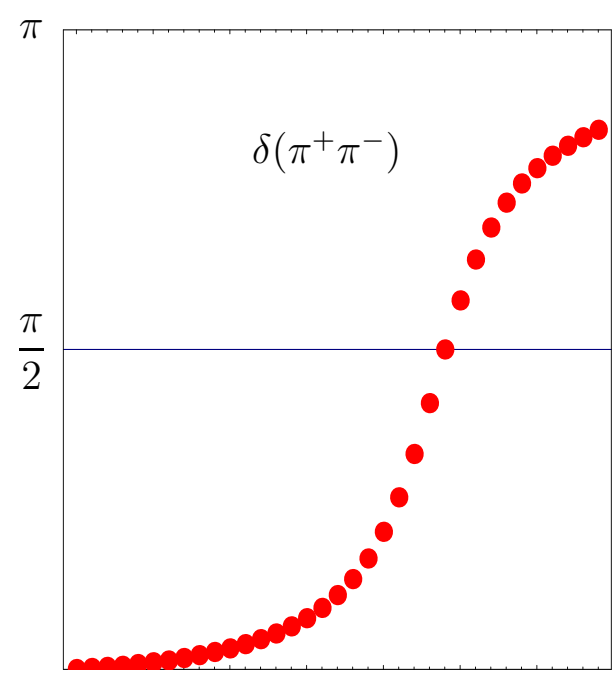

300400500600700800900 $E[\mathrm{MeV}]$

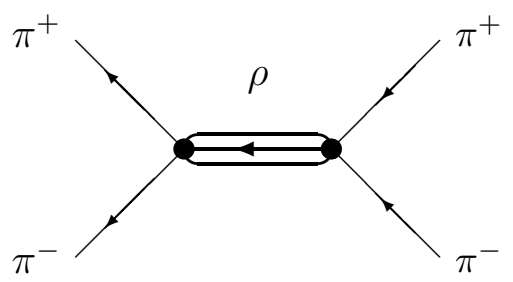

Fig. 2: $\pi^{+} \pi^{-}$p-wave phase-shifts and scattering diagram.

$$
\begin{aligned}
\frac{\mathrm{d} n}{\mathrm{~d} E} & =\frac{\mathrm{d} n^{\text {free }}}{\mathrm{d} E}+\frac{2 j+1}{\pi} \frac{\mathrm{d} \delta_{j}}{\mathrm{~d} E} \\
Z & =\sum_{i} e^{-E_{i} / T}=\int \mathrm{d} E \frac{\mathrm{d} n}{\mathrm{~d} E} e^{-E / T}
\end{aligned}
$$

Since $Z$ determines the equation of state, its low density limit is uniquely given by the scattering phase-shifts. The energy derivatives of the phase-shifts are also responsible for the timedelays discussed in ref. [21] and also for the virial corrections to the Boltzmann collision term recently discussed in ref. [22]. The latter is directly connected to the $B$-term in our generalized kinetic equation (20). The advance of a phase-shift by a value range of $\pi$ across a certain energy window adds one state to the level density and points towards an $s$-channel resonance. An example is the $\rho$-meson in the $p$-wave $\pi^{+} \pi^{-}$scattering channel, fig. 2. In cases, where the resonance couples to one asymptotic channel only, the corresponding phase-shifts relate to the vacuum spectral function $A_{j}(p)$ of that resonance via*

$$
\begin{aligned}
4\left|T_{\mathrm{in}, \text { out }}\right|^{2} & =\frac{\Gamma_{\mathrm{in}}(E) \Gamma_{\mathrm{out}}(E)}{\left(E^{\kappa}-E_{R}^{\kappa}(E)\right)^{2}+\Gamma_{\mathrm{tot}}^{2}(E) / 4} \\
& =4 \sin ^{2} \delta_{j}(E)=A_{j}(E, \vec{p}=0) \Gamma_{\mathrm{tot}}(E) .
\end{aligned}
$$

Here $T_{\mathrm{in}, \text { out }}$ is the corresponding $T$-matrix element. While relation (10) is correct also in the case where many channels couple to the same resonance, relation (111) only holds for the single channel case, where $\Gamma_{\text {in }}=\Gamma_{\text {out }}=\Gamma_{\text {tot }}$. Relation (11) illustrates that the vacuum spectral functions of resonances can almost model-independently be deduced from phase-shift information. In the case of the $\rho$-meson additional information is provided by the pion form factor. Also in the case of two channels coupling to a resonance the energy dependence of phase-shifts of the two scattering channels together with the inelasticity coefficient provide stringent constraints for the spectral function of the resonance [23].

${ }^{*} E$ is the relative c.m. energy and correspondingly the momentum in $A$ vanishes; $\kappa=1$ for non-rel. particles; $\kappa=2$ for relativistic bosons, where $\Gamma(E) / 2 E$ equals the energy dependent decay width and $E_{R}^{2}(E)=m_{R}^{2}+\vec{p}^{2}+\operatorname{Re} \Sigma(p)$. 


\section{The $\rho$-meson in dense matter}

An an example I like to discuss the properties of the $\rho$-meson and the consequences for the decay into di-leptons. The exact production rate of di-leptons is given by the following formula

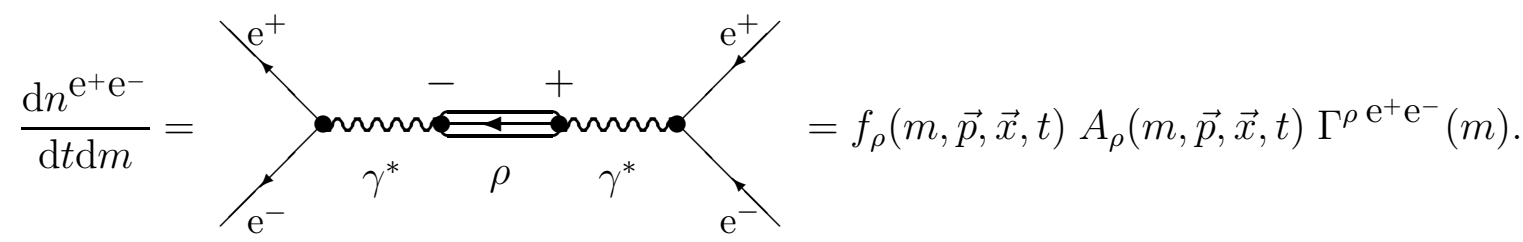

Here $\Gamma^{\rho} \mathrm{e}^{+} \mathrm{e}^{-}(m) \propto 1 / m^{2}$ is the mass-dependent electromagnetic decay rate of the $\rho$-meson into the di-electron channel. The phase-space distribution $f_{\rho}(m, \vec{p}, \vec{x}, t)$ and the spectral function $A_{\rho}(m, \vec{p}, \vec{x}, t)$ define the properties of the $\rho$-meson at space-time point $\vec{x}, t$. Both quantities are in principle to be determined dynamically by an appropriate transport model. However till to-date the spectral functions are not treated dynamically in most of the present transport models. Rather one employs on-shell $\delta$-functions for all stable particles and spectral functions fixed to the vacuum shape for resonances.

As an illustration the model case is discussed, where the $\rho$-meson just strongly couples to two channels: naturally the $\pi^{+} \pi^{-}$channel and to the $\pi N \leftrightarrow \rho N$ channels relevant at finite nuclear densities. The latter component is representative for all channels contributing to the so-called direct $\rho$ in transport codes. For a first orientation the equilibrium properties are discussed. Admittedly by far more sophisticated and in parts unitary consistent equilibrium calculations have already be presented in the literature, e.g. [24-28]. It is not the point to compete with them at this place. Rather we try to give a detailed analysis in simple terms with the aim to discuss the consequences for the implementation of such resonance processes into dynamical transport simulation codes.

Both considered processes add to the total width of the $\rho$-meson

$$
\Gamma_{\text {tot }}(m, \vec{p})=\Gamma_{\rho \rightarrow \pi^{+} \pi^{-}}(m, \vec{p})+\Gamma_{\rho \rightarrow \pi N N^{-1}}(m, \vec{p})
$$

and the equilibrium spectral function then results from the cuts of the two diagrams

$$
A_{\rho}(m, \vec{p})=\Longleftrightarrow\left(m_{\pi^{-}}^{\pi^{+}} \Longleftrightarrow \frac{\Gamma_{\rho \pi^{+} \pi^{-}}+\Gamma_{\rho \pi N N^{-1}}}{\left(m^{2}-m_{\rho}^{2}-\operatorname{Re} \Sigma\right)^{2}+\Gamma_{\mathrm{tot}}^{2} / 4} .\right.
$$

In principle both diagrams have to be calculated by fully self consistent propagators, i.e. with corresponding widths for all particles involved. This formidable task has not been done yet. Using micro-reversibility and the properties of thermal distributions the two terms in (14) contributing to the di-lepton yield (12) can indeed approximately be reformulated as the thermal average of a $\pi^{+} \pi^{-} \rightarrow \rho \rightarrow \mathrm{e}^{+} \mathrm{e}^{-}$-annihilation process and a $\pi N \rightarrow \rho N \rightarrow \mathrm{e}^{+} \mathrm{e}^{-} N$-scattering process, i.e.

$$
\frac{\mathrm{d} n^{\mathrm{e}^{+} \mathrm{e}^{-}}}{\mathrm{d} m \mathrm{~d} t} \propto\left\langle f_{\pi^{+}} f_{\pi^{-}} v_{\pi \pi} \sigma\left(\pi^{+} \pi^{-} \rightarrow \rho \rightarrow \mathrm{e}^{+} \mathrm{e}^{-}\right)+f_{\pi} f_{N} v_{\pi N} \sigma\left(\pi N \rightarrow \rho N \rightarrow \mathrm{e}^{+} \mathrm{e}^{-} N\right)\right\rangle_{T}
$$

However, the important fact to be noticed is that in order to preserve unitarity the corresponding cross sections are no longer the free ones, as given by the vacuum decay width in the denominator, but rather involve the medium dependent total width (13). This illustrates in simple terms that rates of broad resonances can no longer simply be added in a perturbative way. Since it concerns a coupled channel problem there is a cross talk between the different channels to the extent that the common resonance propagator attains the total width arising from all partial widths feeding and depopulating the resonance. While a perturbative treatment with free cross sections in (15) would enhance the yield at resonance, $m=m_{\rho}$, if a channel is added, c.f. fig. 2 left part, the correct treatment (14) even inverts the trend and indeed depletes the yield at resonance, right part in fig. 2. Furthermore one sees that only the total yield involves the spectral function, while any partial cross section only refers to that partial term with the corresponding partial width in the numerator! Unfortunately so far all these facts have been ignored or even overlooked in the present transport treatment of broad resonances. 
Di-lepton rates from thermal $\rho$-mesons $(T=110 \mathrm{MeV})$
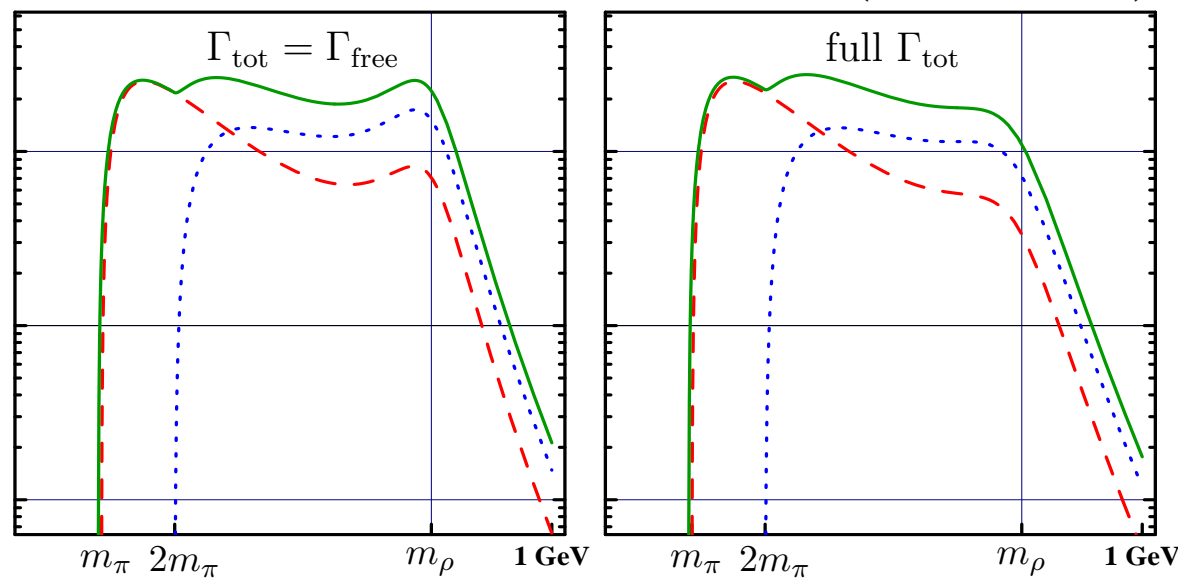

Fig. 3: $\mathrm{e}^{+} \mathrm{e}^{-}$rates (arb. units) as a function of the invariant pair mass $m$ at $T=110 \mathrm{MeV}$ from $\pi^{+} \pi^{-}$annihilation (dotted line) and direct $\rho$-meson contribution (dashed line), the full line gives the sum of both contributions. Left part: using the free cross section recipe, i.e. with $\Gamma_{\text {tot }}=\Gamma_{\rho \pi^{+} \pi^{-}}$; right part for the correct partial rates (14). The calculation are done with $\Gamma_{\rho \leftrightarrow \pi \pi}\left(m_{\rho}\right) / 2 m_{\rho}=150 \mathrm{MeV}$ and $\Gamma_{\rho \leftrightarrow \pi N N^{-1}}\left(m_{\rho}\right) / 2 m_{\rho}=70 \mathrm{MeV}$.
Quasi-free $\pi N$ collisions and spectral function

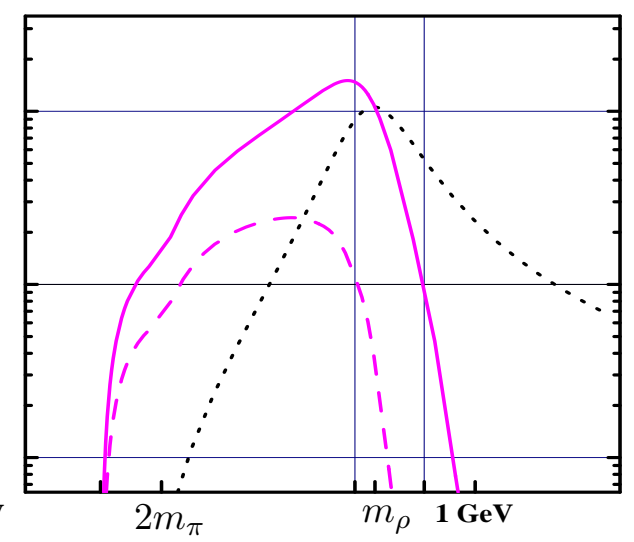

Fig. 4: Fermi motion averaged $\pi N \rightarrow$ $\rho N \rightarrow \mathrm{e}^{+} \mathrm{e}^{-} N$ cross sections at pion beam momenta of 1 and $1.3 \mathrm{GeV} / \mathrm{c}$ (dashed and full curve) as a function of invariant pair mass $m$. The dotted line gives the spectral function used here and in fig. 3 .

Compared to the spectral function (dotted line in fig. 4) both thermal components in fig. 3 show a significant enhancement on the low mass side and a strong depletion at high masses due to the thermal weight $f \propto \exp \left(-p_{0} / T\right)$ in the rate (12). A similar effect is seen in genuine non-equilibrium processes like the di-lepton yield resulting from Fermi-motion averaged $\pi N \rightarrow \rho N$ scattering, fig. 4. The latter is representive for the first chance collision in a $\pi A$ reaction and shows a behavior significantly different from that obtained in refs. [30]. For orientation the sub-threshold conditions for the two beam momenta are given by the vertical lines in fig. 4 !

Much of the physics can already be discussed by observing that the partial widths are essentially given by the type of coupling ( $s$ or $p$-wave, $l=0,1$ ) and the phase space available for the decay channel. For point-like couplings and two-body phase space or approximately in the case of one light particle among only heavy ones in the decay channel (e.g. for $\pi N N^{-1}$ ) one finds

$$
\Gamma_{c}(m) \propto m p_{\mathrm{cm}}\left(\frac{p_{\mathrm{cm}}}{m}\right)^{l} \quad \text { with } \quad p_{\mathrm{cm}} \propto \sqrt{m^{2}-s_{\mathrm{thr}}} ;\left\{\begin{array}{ll}
s_{\mathrm{thr}}=4 m_{\pi}^{2}, & l=1 \text { for } c=\{\rho \leftrightarrow \pi \pi\} \\
s_{\mathrm{thr}}=m_{\pi}^{2}, & l=1 \text { for } c=\left\{\rho \leftrightarrow \pi N N^{-1}\right.
\end{array}\right\}
$$

In the $\pi \pi$ case the corresponding strength is approximately given by the vacuum decay, while it depends on the nuclear density in the $\rho N \leftrightarrow \pi N$ case. The simple phase-space behavior (16) suggests that far away from all thresholds $\left(m^{2} \gg s_{\text {thr }}\right)$ the ratio $\langle\pi \pi$-annihilation $\rangle /\langle$ direct $\rho\rangle$ of the two components should result to a fairly smooth and almost constant function of $m$, e.g. for $m>500 \mathrm{MeV}$. This kinematical constraint is nicely confirmed by some calculations, e.g. of ref. [29], however, no such behavior is seen in the direct $\rho$-mesons so far computed in refs. [30, 31] [1.

For completeness and as a stimulus for improvements the discussed defects in some of presently used transport treatments of broad resonances (vector mesons in particular) are listed below. The last column gives an estimate of multiplicative factors needed to restore the defect $\left(m_{R}\right.$ is the resonance mass and $T^{*}$ (between 70 and $120 \mathrm{MeV}$ ) is a typical slope parameter for the corresponding beam energy). Many of the points are known and trivial, and can trivially be implemented. They are just of kinematical origin. However the associated defects are by no means minor. Rather they ignore essential features of the dynamics with consequences already on the qualitative level and affect the spectra by far more than any of the currently discussed im-medium effects, e.g. of the $\rho$-meson.

${ }^{\dagger}$ In refs. 30, 31 the direct $\rho$ component appears almost like the spectral function itself, i.e. untouched from any phase-space constraints which come in through the distributions $f_{\rho}(X, p)$. The latter favour low masses and deplete the high mass components! In fact rather than being almost constant the ratios $\langle\pi \pi\rangle /\langle$ direct $\rho\rangle$ exhibit an exponential behavior $\exp \left(-m / T^{*}\right)$ for $m>500 \mathrm{MeV}$ with $T^{*}$ between $70-110 \mathrm{MeV}$ depending on beam energy, pointing towards a major deficiency in the account of phase-space constraints for the direct $\rho$-meson component in these calculations. 


\section{List of defects in some of the transport codes}

[a] The differential mass information contained in the distribution functions $f(X, p)=f(X, m, \vec{p})$ of resonances is ignored and only the integrated total number is evaluated as a function of space-time (direct $\rho$ in refs. [30, 31]).

[b] Except for the $\pi^{+} \pi^{-} \rightarrow \mathrm{e}^{+} \mathrm{e}^{-}$case most resonance production cross sections are parametrized such that they vanishes for $\sqrt{s}$ values below the nominal threshold, e.g. below $m_{N}+m_{\rho}$ in the case $\pi N \rightarrow \rho N$. This violates detailed balance, since broad resonances can decay for $m<m_{R}$.

[c] In partial cross sections leading to a resonance the randomly chosen mass is normally selected according to the spectral function. This is not correct since the corresponding partial width in the numerator of (14) has to be considered.

[d] Different partial cross sections are simply added without adjusting the total width in the resonance propagator accordingly. This violates unitarity.

[e] The Monte Carlo implementation of selecting the random mass $m$ of the resonance (item $[\mathrm{c}]$ ) is sometimes falsely implemented, namely ignoring the kinetic phase-space of genuine multi-particle final state configurations, e.g. in $\pi N \rightarrow \rho N$. Applies also to the $\Delta$-resonance, e.g. for $N N \rightarrow N \Delta$.

[f] For the electromagnetic decay of vector mesons some authors use a mass independent decay rate, e.g. $\Gamma_{\rho \rightarrow \mathrm{e}^{+} \mathrm{e}^{-}} / m=$ const., rather than that resulting from vector dominance and QED with $\Gamma_{\rho \rightarrow \mathrm{e}^{+} \mathrm{e}^{-}} \propto 1 / m^{2}$. restoring factor

$\exp \left(-\left(m-m_{R}\right) / T^{*}\right)$

(factor 10 or more at $m=500 \mathrm{MeV}$ for $\rho$ )

misses yield for

$m<m_{R}$

changes shape

$\left(\Gamma_{\text {free }} / \Gamma_{r m t o t}\right)^{2}$

at $m=m_{R}$

proportional to

$\left(s\left(\sqrt{s}-m-m_{N}\right)\right)^{1 / 2}$

for two-body final state

$\left(m_{R} / m\right)^{3}$

\section{6 - $\quad$-derivable approximations}

The preceding section has shown that one needs a transport scheme adapted for broad resonances. Besides the conservation laws it should comply with requirements of unitarity and detailed balance. A practical suggestion has been given in ref. [4] in terms of cross section prescriptions. However this picture is tied to the concept of asymptotic states and therefore not well suited for the general case, in particular if more than one channel feeds into a broad resonance. Therefore we suggest to revive the so-called $\Phi$-derivable scheme, originally proposed by Baym [11] on the basis of a formulation of the generating functional or partition sum given by Luttinger, Ward [32], and later reformulated in terms of path-integrals [33]. This functional can be generalized to the real time case (for details see [16]) with the diagrammatic representationf

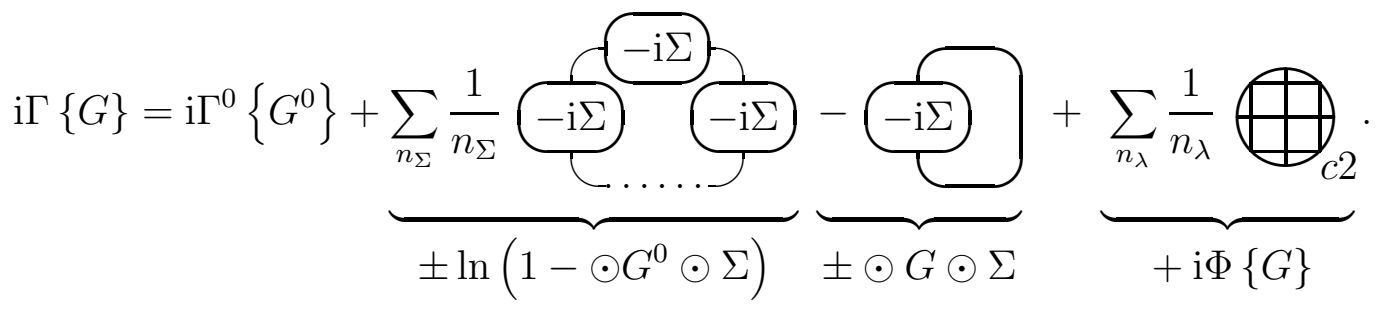

Thereby the key quantity is the auxiliary functional $\Phi$ given by two-particle irreducible vacuum diagrams. It solely depends on fully re-summed, i.e. self consistently generated propagators $G(x, y)$ (thick lines). The consistency is provided by the fact that $\Phi$ is the generating functional for the re-summed self-energy $\Sigma(x, y)$ via functional variation of $\Phi$ with respect to any propagator $G(y, x)$, i.e.

$$
-\mathrm{i} \Sigma=\mp \delta \mathrm{i} \Phi / \delta \mathrm{i} G \text {. }
$$

The Dyson equations of motion directly follow from the stationarity condition of $\Gamma$ (17) with respect to variations of $G$ on the contour

$$
\delta \Gamma\{G\} / \delta G=0, \quad \text { (Dyson eq.) }
$$

$\ddagger n_{\Sigma}$ counts the number of self-energy $\Sigma$-insertions in the ring diagrams, while for the closed diagram of $\Phi$ the value $n_{\lambda}$ counts the number of vertices building up the functional $\Phi$.

$\S_{\text {an }}$ extension to include classical fields or condensates into the scheme is presented in ref. [16] 
In graphical terms, the variation (18) with respect to $G$ is realized by opening a propagator line in all diagrams of $\Phi$. The resulting set of thus opened diagrams must then be that of proper skeleton diagrams of $\Sigma$ in terms of full propagators, i.e. void of any self-energy insertions. As a consequence, the $\Phi$-diagrams have to be two-particle irreducible (label $c 2$ ), i.e. they cannot be decomposed into two pieces by cutting two propagator lines.

The clue is that truncating the auxiliary functional $\Phi$ to a limited subset of diagrams leads to a self consistent, i.e closed, approximation scheme. Thereby the approximate forms of $\Phi^{\text {(appr.) }}$ define effective theories, where $\Phi^{\text {(appr.) }}$ serves as a generating functional for the approximate self-energies $\Sigma^{\text {(appr.) }}(x, y)$ through relation (18), which then enter as driving terms for the Dyson equations of the different species in the system. As Baym [11] has shown such a $\Phi$-derivable approximation is conserving for all conservation laws related to the global symmetries of the original theory and at the same time thermodynamically consistent. The latter automatically implies correct detailed balance relations between the various transport processes. For multicomponent systems it leads to a actio = reactio principle. This implies that the properties of one species are not changed by the interaction with other species without affecting the properties of the latter ones, too. The $\Phi$-derivable scheme offers a natural and consistent way to account for this principle. Some thermodynamic examples have been considered recently, e.g., for the interacting $\pi N \Delta$ system [9] and for a relativistic QED plasma [34.

\section{Generalized Kinetic Equation}

In terms of the kinetic notation (4) and in the first gradient approximation the generalized kinetic equation for $F$ takes the form

$$
\mathcal{D} F(X, p)=B_{\text {in }}(X, p)+C(X, p)
$$

with the drift term determined from the "mass" function (c.f. (白))

$$
M(X, p)=M_{0}(p)-\operatorname{Re} \Sigma^{R}(X, p)
$$

through the Poisson bracket $\mathcal{D} F \equiv\{M, F\}$. The explicit form of the differential drift operator reads

$$
\mathcal{D}=\left(v_{\mu}-\frac{\partial \operatorname{Re} \Sigma^{R}}{\partial p^{\mu}}\right) \partial_{X}^{\mu}+\frac{\partial \operatorname{Re} \Sigma^{R}}{\partial X^{\mu}} \frac{\partial}{\partial p_{\mu}}, \quad \text { with } \quad v^{\mu}=\frac{\partial M_{0}(p)}{\partial p_{\mu}}= \begin{cases}(1, \vec{p} / m) & \text { non-rel. } \\ 2 p^{\mu} & \text { rel. bosons. }\end{cases}
$$

The two other terms in (20), $B_{\text {in }}(X, p)$ and $C(X, p)$, are a fluctuation term and the collision term, respectively

$$
B_{\text {in }}=\left\{\Gamma_{\text {in }}, \operatorname{Re} G^{R}\right\}, \quad C(X, p)=\Gamma_{\text {in }}(X, p) \widetilde{F}(X, p)-\Gamma_{\text {out }}(X, p) F(X, p), .
$$

Here the reduced gain and loss rates and total width of the collision integral are

$$
\begin{gathered}
\Gamma_{\text {in }}(X, p)=\mp \mathrm{i} \Sigma^{-+}(X, p), \quad \Gamma_{\text {out }}(X, p)=\mathrm{i} \Sigma^{+-}(X, p), \\
\Gamma(X, p) \equiv-2 \operatorname{Im} \Sigma^{R}(X, p)=\Gamma_{\text {out }}(X, p) \pm \Gamma_{\text {in }}(X, p) .
\end{gathered}
$$

The combination opposite to (25) determines the fluctuations

$$
I(X, p)=\Gamma_{\text {in }}(X, p) \mp \Gamma_{\text {out }}(X, p) .
$$

We need still one more equation, which in fact can be provided by the retarded Dyson equation. In first order gradient approximation the latter is completely solved algebraically [5]

$$
G^{R}=\frac{1}{M(X, p)+\mathrm{i} \Gamma(X, p) / 2} \quad \Rightarrow \quad A(X, p)=\frac{\Gamma(X, p)}{M^{2}(X, p)+\Gamma^{2}(X, p) / 4}
$$

Canonical equal-time (anti) commutation relations for (fermionic) bosonic field operators provide the standard sum-rule for the spectral function. 
We now provide a physical interpretation of the various terms in the generalized kinetic equation (20). The drift term $\mathcal{D} F$ on the l.h.s. of eq. (20) is the usual kinetic drift term including the corrections from the self-consistent field $\operatorname{Re} \Sigma^{R}$ into the convective transfer of real and also virtual particles. For the collision-less case $C=B=0$, i.e. $\mathcal{D} F=0$ (Vlasov equation), the quasi-linear first order differential operator $\mathcal{D}$ defines characteristic curves. They are the standard classical paths in the Vlasov case. Thereby the four-phase-space probability $F(X, p)$ is conserved along these paths. The formulation in terms of a Poisson bracket in four dimensions implies a generalized Liouville theorem. For the collisional case both, the collision term $C$ and the fluctuation term $B$ change the phase-space probabilities of the "generalized" particles during their propagation along the "generalized" classical paths given by $\mathcal{D}$. We use the term "generalized" in order to emphasize that particles are no longer bound to their mass-shell, $M=0$, during propagation due to the collision term, i.e. due decay, creation or scattering processes.

The r.h.s. of eq. (20) specifies the collision term $C$ in terms of gain and loss terms, which also can account for multi-particle processes. Since $F$ includes a factor $A$, the $C$ term further deviates from the standard Boltzmann-type form in as much that it is multiplied by the spectral function $A$, which accounts for the finite width of the particles.

The additional Poisson-bracket term

$$
B_{\text {in }}=\left\{\Gamma_{\text {in }}, \operatorname{Re} G^{R}\right\}=\frac{M^{2}-\Gamma^{2} / 4}{\left(M^{2}+\Gamma^{2} / 4\right)^{2}} \mathcal{D} \Gamma_{\text {in }}+\frac{M \Gamma}{\left(M^{2}+\Gamma^{2} / 4\right)^{2}}\left\{\Gamma_{\text {in }}, \Gamma_{\text {out }}\right\}
$$

is special. It contains genuine contributions from the finite mass width of the particles and describes the response of the surrounding matter due to fluctuations. This can be seen from the conservation laws discussed below. In particular the first term in (28) gives rise to a back-flow component of the surrounding matter. It restores the Noether currents to be conserved rather than the intuitively expected sum of convective currents arising from the convective $\mathcal{D} F$ terms in (20). The second term of (28) gives no contribution in the quasi-particle limit of small damping width limit and represents a specific off mass-shell response due to fluctuations, c.f. [35, 14]. In the low density and quasi-particle limit the $B_{\text {in }}$ term provides the virial corrections to the Boltzmann collision term [22].

\section{Conservations of the Current and Energy-Momentum}

The global symmetries of $\Phi$ provide conservation laws such as the conservation of charge and energymomentum. The corresponding Noether charge current and Noether energy-momentum tensor result to the following expressions, c.f. [16],

$$
\begin{aligned}
j^{\mu}(X) & =\frac{e}{2} \operatorname{Tr} \int \frac{\mathrm{d}^{4} p}{(2 \pi)^{4}} v^{\mu}(F(X, p) \mp \widetilde{F}(X, p)) \\
\Theta^{\mu \nu}(X) & =\frac{1}{2} \operatorname{Tr} \int \frac{\mathrm{d}^{4} p}{(2 \pi)^{4}} v^{\mu} p^{\nu}(F(X, p) \mp \widetilde{F}(X, p))+g^{\mu \nu}\left(\mathcal{E}^{\operatorname{int}}(X)-\mathcal{E}^{\text {pot }}(X)\right) .
\end{aligned}
$$

Here

$$
\mathcal{E}^{\text {int }}(X)=\left\langle-\widehat{\mathcal{L}}^{\text {int }}(X)\right\rangle=\left.\frac{\delta \Phi}{\delta \lambda(x)}\right|_{\lambda=1}, \quad \mathcal{E}^{\text {pot }}=\frac{1}{2} \operatorname{Tr} \int \frac{\mathrm{d}^{4} p}{(2 \pi)^{4}}\left[\operatorname{Re} \Sigma^{R}(F \mp \widetilde{F})+\operatorname{Re} G^{R}\left(\Gamma_{\text {in }} \mp \Gamma_{\text {out }}\right)\right]
$$

are the densities of the interaction energy and the potential energy, respectively. The first term of $\mathcal{E}^{\text {pot }}$ complies with quasi-particle expectations, namely mean potential times density, the second term displays the role of fluctuations $I=\Gamma_{\text {in }} \mp \Gamma_{\text {out }}$ in the potential energy density. This fluctuation term precisely arises form the $B$-term in the kinetic eq. (20), discussed around eq. (28). It restores that the Noether expressions (29) are indeed the exactly conserved quantities. In this compensation we see the essential role of the fluctuation term in the generalized kinetic equation. Dropping or approximating this term would spoil the conservation laws. Indeed, both expressions in (29) comply exactly with the generalized kinetic equation (20), i.e. they are exact integrals of the generalized kinetic equations of 
motion within the $\Phi$-derivable scheme. Memory effects and the formulation of a kinetic entropy can likewise be addressed [14.

Acknowledgement: Much of the material presented is due a very stimulating collaboration with Y. Ivanov and D. Voskresensky. The author further acknowledges encouraging discussions with P. Danielewicz, B. Friman, H. van Hees, E. Kolomeitsev, M. Lutz, K. Redlich and W. Weinhold on various aspects of broad resonances and J. Aichelin, S. Bass, E. Bratkowskaya, W. Cassing, C.M. Ko and U. Mosel on some particular features of transport codes.

\section{References}

[1] J. Schwinger, J. Math. Phys, 2 (1961) 407; L. P. Kadanoff and G. Baym, Quantum Statistical Mechanics (Benjamin, 1962); L. M. Keldysh, ZhETF 47 (1964) 1515; in Engl. translation Sov. Phys. JETP20 (1965) 1018.

[2] P. Danielewicz, Ann. Phys. (N. Y.) 152 (1984) 239

[3] N. P. Landsmann, Phys. Rev. Lett. 60 (1988) 1990; Ann. Phys. 186 (1988) 141.

[4] P. Danielewicz, G. Bertsch, Nucl. Phys. A533 (1991) 712.

[5] W. Botermans and R. Malfliet, Phys. Rep. 198 (1990) 115.

[6] M. Herrmann, B. L. Friman, W. Nörenberg, Nucl. Phys. A560 (1993) 411.

[7] P. A. Henning, Phys. Rep. C 253 (1995) 235; Nucl. Phys. A 582 (1995) 633.

[8] E. Quack, P. A. Henning, GSI-95-29; Phys. Rev. Lett. in print; GSI-95-42.

[9] W. Weinhold, Diploma thesis, GSI 1995; W. Weinhold, B. L. Friman, W. Nörenberg, Acta Phys. Pol. 27, Phys. Lett. B 433 (1998) 236.

[10] J. Knoll and D. N. Voskresensky, Ann. Phys 249 (1996) 532;

a condensed account of this work is published in Phys. Lett. B 351 (1995) 43.

[11] G. Baym, Phys. Rev. 127 (1962) 1391.

[12] C. Greiner, K. Wagner, P.G. Reinhard, Phys.Rev. C49 (1994) 1693.

[13] J. Knoll et al., Proceedings of the Thermal Field Theory workshop, TFT 98, Regensburg 1998, hep-ph/9809419

[14] Y. Ivanov, J. Knoll, D. Voskresenski, in preparation

[15] E. M. Lifshitz and L. P. Pitaevskii, "Physical Kinetics" Nauka, 1979; Pergamon press, 1981.

[16] Yu. B. Ivanov, J. Knoll and D. N. Voskresenski, GSI-preprint-98-34, hep-ph/9807351.

[17] E. Beth, G.E. Uhlenbeck, Physica 4 (1937) 915

[18] K. Huang, "Statistical Mechanics", Wiley, New York (1963)

[19] R. Dashen, S. Ma, H.J. Bernstein, Phys.Rev. 187 (1969) 345;

[20] A.Z. Mekjian, Phys.Rev. C 17 (1978) 1051;

[21] P. Danielewicz and S. Pratt, Phys.Rev. C53 (1996) 249

[22] V. Špička, P. Lipavský, K. Morawetz, Phys. Lett. A240 (1998) 160;

[23] W. Weinhold, Ph-D thesis, TU-Darmstadt, 1998

[24] S. Leupold, U. Mosel, Phys. Rev. C 58 (1998) 2939; U. Mosel, these proceedings;

[25] R. Rapp, G. Chanfray, J. Wambach, Nucl. Phys. A 617 (1997)472;

[26] F. Klingl, N. Kaiser, W. Weise, Nucl. Phys. A 624 (1997) 527;

[27] B.L. Friman, H.-J. Pirner, Nucl. Phys. A 617 (1997) 496;

[28] B. Friman, M. Lutz and G. Wolf, GSI-Preprint-98-63, nucl-th/9811040;

[29] W.S. Chung, C.M. Ko, G.Q. Li, Nucl. Phys. A 641 (1998) 357;

[30] c.f. Figs. 3 and 4 in W. Cassing et. al., Phys. Lett. B 396 (1997) 26 or Weidmann et al., nuclth/9711004;

[31] c.f. Figs. 2 - 4 in W. Cassing et. al., Phys. Rev. C57 (1997) 916, or , and corresponding earlier references therein;

[32] J. M. Luttinger and J. C. Ward, Phys. Rev. 118 (1960) 1417.

[33] J.M. Cornwall, R. Jackiw and E. Tomboulis, Phys. Rev. D 10 (1974) 2428;

[34] B. Vanderheyden and G. Baym, hep-ph/9803300;

[35] V. Spicka and P. Lipavsky, Phys. Rev. Lett. 73 (1994) 3439; Phys. Rev. B52 (1995) 14615. 